Bolm Inst. oceanogr., S Paulo, 42(1/2):99-111, 1994

\title{
Macrozoobentos da Praia do Codó (Ubatuba, SP) e a presença de Halodule wrightii Ascherson
}

\author{
Thais Navajas CORBISIER
}

Instituto Oceanográfico da Universidade de São Paulo

(Caixa Postal 66149, 053149-970 Sāo Paulo, SP, Brasil)

- Abstract: A year's survey conducted at a seagrass (Halodule wrightii Ascherson) bed and an adjacent bare sand area, on Cod6 Beach, Ubatuba, southern Brazil, showed that the macrobenthic species composition was different at the two habitats. Surface deposit feeder infaunal polychaetes were the dominant species in the Halodule site and omnivorous/carnivorous and filter feeder polychaetes, in the bare sand. These differences could be explained mainly by the characteristics of the sediment and the stability of the bottom and, secondly, by the presence of Halodule. The presence of vegetation has no effect on density of macrobenthos, probably due to the small size of Halodule plants, which do not support an abundant epifauna. Despite that fact, both number and diversity of species were higher in the seagrass area than in the unvegetated site, as was epifaunal abundance. Significant patterns of temporal fluctuations of macrobenthos abundance and species diversity were not observed, but diversity was the highest in Spring at both habitats. The temporal fluctuations of abundance differed in the two habitats. At the unvegetated site instability of the substrate seems to be responsible for the observed fluctuations in densities. At the Halodule site only light fluctuations occurred, though they did not follow those of macrophyte biomass. Macropredator effects are suggested to be one of the controling factors of macrobenthos density at the vegetated habitat.

- Resumo: Neste trabalho, foi estudada a macrofauna bentônica da franja do infralitoral da Praia do Cod6, Ubatuba, em uma área com a presença da angiosperma marinha Halodule wrightii Ascherson e em área próxima desprovida de vegetaçäo, trimestralmente, no período de dezembro de 1984 a dezembro de 1985. Analisou-se a composição de espécies, a estrutura e a dinâmica da associaçōes da macrofauna e avaliou-se sua relaçẩo com os fatores tipo de şedimento e presença da vegetação. Foram utilizados tubos de PVC de $78,5 \mathrm{~cm}^{2}$ de área de secção, enterrados a $10 \mathrm{~cm}$ no sedimento, e peneira de $0,5 \mathrm{~mm}$ de abertura de malha no estudo da macrofauna. A granulometria, a matéria orgânica no sedimento e o peso seco da vegetação foram analisados. $O$ trabalho mostrou que os habitats diferiram quanto à composição em espécies da macrofauna bentônica. Espécies de poliquetos infaunais comedores de depósito de superfície foram as dominantes na área com Halodule e poliquetos infaunais carnívoros/omnívoros ou filtradores, na área desprovida de vegetação. Estas diferenças puderam ser explicadas principalmente pelas diferenças observadas nas características do sedimento e na estabilidade do fundo e, secundariamente, pela presença ou ausência da vegetação. Não se observou qualquer efeito da presença da vegetação sobre a densidade do macrobentos, provavelmente, em função do pequeno tamanho das plantas que não oferecem substrato para uma epifauna abundante; ainda assim, o número de espécies e a diversidade de espécies, bem como o número de espécies da epifauna, foram maiores na área de Halodule do que na área sem vegetação. Não foi observado um padrāo de variaçāo temporal significativo da abundância e diversidade da macrofauna, mas, em geral, a diversidade foi maior na primavera, nos dois habitats. A variação temporal da abundância diferiu entre os dois locais. $\mathrm{Na}$ área sem vegetação, a maior instabilidade do fundo deve ser a causa das variaçōes observadas. $\mathrm{Na}$ área com Halodule, as flutuaçōes não foram muito pronunciadas e não acompanharam as da biomassa das plantas, que foi mais alta no verāo. Sugere-se que o efeito da predação seja um dos fatores controladores da densidade da macrofauna na área com Halodule.

- Descriptors: zoobenthos, seagrass, Halodule, Ubatuba, Brazil

- Descritores: zoobentos, angiosperma marinha, Halodule, Ubatuba, Brasil 


\section{Introdução}

Pradarias de angiospermas marinhas (seagrasses na língua inglêsa) são sistemas altamente produtivos e que proporcionam habitat e alimento para uma fauna variada, de valor ecológico e comercial. Além disso, provêm matéria orgânica para ciclagem de diversos nutrientes e contribuem significativamente para a estabilizaçāo do sedimento (McRoy \& Hellferich, 1977; Fredette et al., 1990).

Esses sistemas de angiospermas marinhas estão relacionados a uma fauna de invertebrados mais abundante e rica em espécies que habitats próximos, desprovidos de vegetação (Santos \& Simon, 1974; Heck \& Wetstone, 1977; Orth, 1977; Virnstein et al., 1983; Lewis, 1984; Summerson \& Peterson, 1984; Wells et al., 1985; Edgar, 1990a; Wilson, 1990). As razōes apontadas para explicar tal fato, quando da presença de angiospermas, são o aumento a) da quantidade de espaço disponível, b) do número de microhabitats, c) dos recursos alimentares, d) de refúgio contra a predação e e) da estabilização do sedimento, e a reduçāo do hidrodinamismo (Lewis, 1984; Orth et al., 1984); acrescentam-se a esses a seleção ativa de habitats com macrófitas pelas espécies macrofaunais (Bell \& Westoby, 1986) ou o aumento do estabelecimento de larvas planctônicas de invertebrados em pradarias de angiospermas, seja por processos ativos ou passivos (Wilson, 1990).

No Brasil, essas monocotiledôneas podem constituir a vegetaçāo dominante em amplas áreas do litoral ao longo de sua costa, sendo Halodule wrightii Ascherson uma das que apresentam ampla distribuição na costa brasileira (Oliveira et al., 1983). Entretanto, são poucos os estudos sobre a fauna de pradarias de angiospermas no Brasil. Asmus (1984) estudou a estrutura da comunidade associada a Ruppia maritima na Lagoa dos Patos, Rio Grande do Sul, e Corbisier et al. (1986) iniciaram um estudo sobre a ecologia de um banco de Halodule emarginata em São Sebastiāo, São Paulo.

Este trabalho é um estudo comparativo da macrofauna bentônica de uma área com a presença de Halodule wrightii e de outra adjacente, desprovida de vegetação, na Praia do Cod6, situada no Saco da Ribeira, Ubatuba, SP, no período de um ano. Os objetivos foram estudar as associaçōes de espécies da macrofauna bentônica dos dois locais, quanto a sua composiçāo específica, estrutura e dinâmica, e avaliar sua relação com fatores como as características do sedimento e a presença de Halodule.

\section{Material e métodos}

Área do estudo - Os dois locais amostrados estão situados na Praia do Codó a noroeste do Saco da Ribeira,
Enseada do Flamengo, Ubatuba, SP (23⒊' S-4507.4' W) (Fig. 1). O Saco da Ribeira é raso, apresentando $5 \mathrm{~m}$ de profundidade no seu ponto mais profundo, e os locais estudados situam-se no nível - 0,3 , na franja do infralitoral. A planta é encontrada formando uma faixa estreita (cerca de $10 \mathrm{~m}$ ) à volta do Saco da Ribeira. Um dos locais de coleta foi posicionado onde as plantas atingiam sua maior biomassa ( média mensal de $42 \mathrm{~g}$ peso seco total. $\mathrm{m}^{-2}$, Corbisier, 1989); o outro, em área adjacente sem vegetação, distante $40 \mathrm{~m}$ do primeiro, mas submetida a uma maior turbulência hidrodinâmica devido à presença de matacōes ao seu redor, fato esse evidenciado pela presença ocasional de marcas onduladas ("ripple marks").
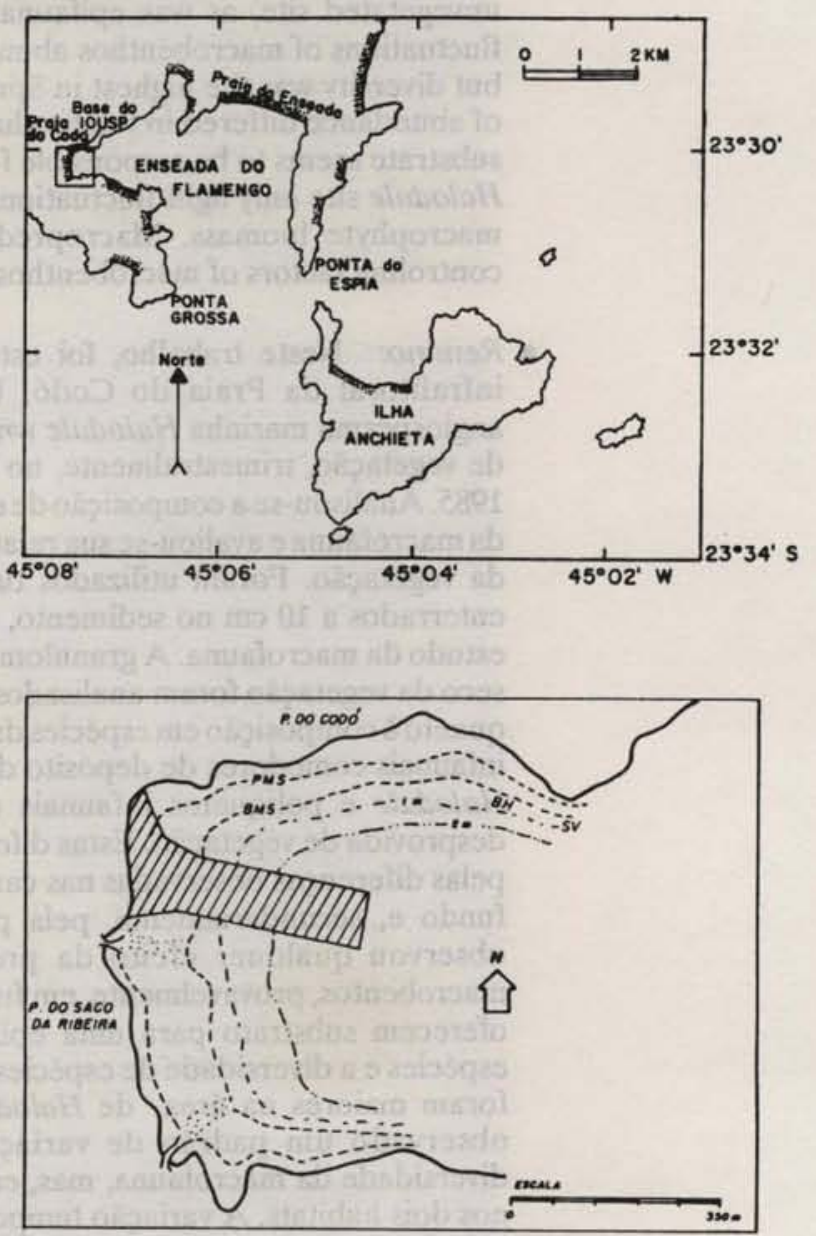

Fig. 1. Mapa da região do estudo, com a localização da Praia do Codó e dos locais de coleta. $\mathrm{BH}$ - banco de Halodule; SV - área sem vegetação. 
A água da enseada é oligotrófica (Teixeira, 1979). A salinidade e a temperatura médias da água refletem normalmente aquelas das enseadas próximas, tendo variado de 30.1 a $34.8 \%$ e 20.4 a $27.2^{\circ} \mathrm{C}$, respectivamente, no período do estudo, na Enseada de Ubatuba, vizinha à do Flamengo (Fig. 2). A salinidade decresceu naquela enseada nos meses de verão, quando a pluviosidade e a temperatura foram maiores (Fig. 2). As marés são semi-diurnase a amplitude de maré é de 1,6 m (nível médio do mar 0,7 m) (Mesquita e Harari, 1983).

Amostragem - As coletas foram realizadas trimestralmente, de dezembro de 1984 a dezembro de 1985. Em cada ocasiāo e habitat, quatro amostras de sedimento foram retiradas, aleatóriamente, de duas áreas de $1 \mathrm{~m}^{2}$ cada, com tubos de PVC de $78,5 \mathrm{~cm}^{2}$ de área de secçāo, enterrados a $10 \mathrm{~cm}$ no sedimento. Os tubos tinham $10 \mathrm{~cm}$ de diâmetro, $60 \mathrm{~cm}$ de altura e tela de nylon de 0,5 $\mathrm{mm}$ de abertura de malha no topo. Com esta metodologia, foram coletados os organismos da epifauna e da infauna. As amostras foram peneiradas separadamente em tela de abertura de malha de $0,5 \mathrm{~mm}$; o sedimento remanescente foi preservado em álcool $70 \%$ e, posteriormente, triado sob estereomicroscópio. Os organismos foram identificados e contados.

Nas mesmas ocasiōes, duas amostras de sedimento foram coletadas com o mesmo tubo descrito acima, com a finalidade de se analisar a sua granulometria e o conterido de matéria orgânica. A granulometria foi analisada pelos métodos de peneiramento e pipetagem (Suguio, 1973). A fim de estimar a porcentagem total de matéria orgânica, uma alíquota de $1 \mathrm{~g}$ foi retirada da amostra seca, antes da sua lavagem, e foi oxidada com adiçâo de $\mathrm{H}_{2} \mathrm{O}_{2} 30 \%$ num béquer sobre uma placa aquecida (Gross, 1971).

As plantas presentes em cada amostra foram retiradas, lavadas sobre peneira de abertura de malha de $0,5 \mathrm{~mm}$, e examinadas sob estereomicroscópio para retirada de fauna associada. Posteriormente, foram secas em estufa a $60^{\circ} \mathrm{C}$ a fim de se determinar seu peso seco.

Análises - As espécies foram classificadas como constantes, acessórias e raras de acordo com sua frequeência nas amostras (Dajoz, 1973). Os organismos foram classificados como epifauna e infauna de acordo com o critério de Summerson \& Peterson (1984). A definição dos grupos funcionais foi baseada em informaçōes disponíveis nos trabalhos de Bousfield (1973), Fauchald \& Jumars (1979), Stoner (1980), Lopes (1983) e Williams (1984).

A estrutura das associaçōes de espécies foi avaliada comparando-se o número de indivíduos $(\mathrm{N})$, a riqueza $\mathrm{em}$ espécies (S, número de espécies), os índices de diversidade de Shanon $\left(H^{\prime}, \log _{2}\right)$ e de equitatividade de Pielou $\left(\mathrm{J}^{\prime}\right)$ por amostra (Zar, 1984), bem como as razōes epifauna sobre a fauna total e poliquetos com mobilidade sobre o total de poliquetos, baseando-se no conceito de grupos funcionais.

A fim de testar as diferenças entre os locais, quanto aos fatores ambientais e quanto à estrutura das associaçōes, foram utilizados os testes não paramétricos de Wilcoxon (para duas amostras relacionadas) e de Mann-Whitney
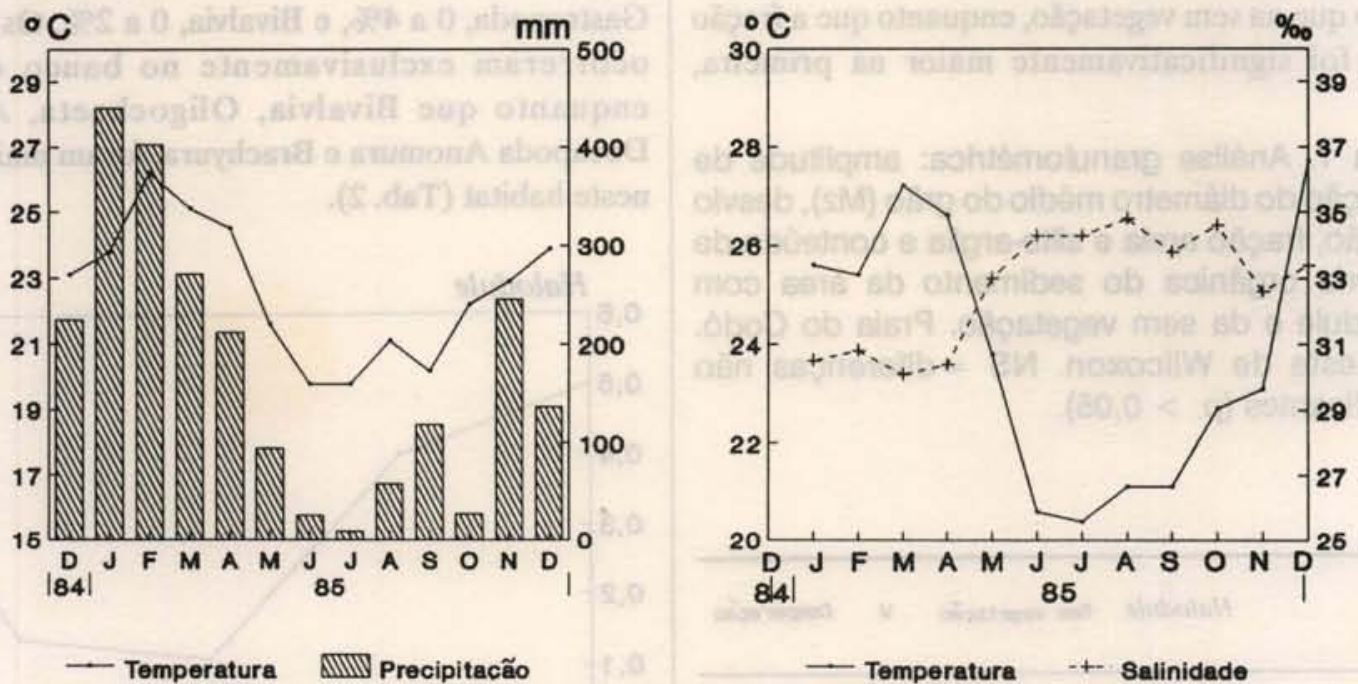

Fig. 2. Variação temporal da média mensal (A) da temperatura do $\operatorname{ar}\left({ }^{\circ} \mathrm{C}\right)$ e da precipitação atmosférica $(\mathrm{mm})$, em Ubatuba ${ }^{1}$, e $(B)$ da temperatura $\left({ }^{\circ} \mathrm{C}\right)$ e salinidade $(\% \circ)$ da água de superfície da Enseada de Ubatuba ${ }^{2}$, no período do estudo. 1 - Dados fornecidos pela Secção de Meteorologia do IOUSP.

2 - Dados fornecidos pelo Instituto de Pesca da Secretaria da Agricultura, Seção Ubatuba. 
(para duas amostras independentes) (Zar, 1984). As variaçōes temporais foram testadas através da análise de variância de postos de Kruskal- Wallis; no caso de diferenças significativas, foram feitas comparações múltiplas utilizando-se o teste de Dunn (Gibbons, 1976).

As associações faunísticas dos dois locais foram analisadas usando-se o índice de dissimilaridade de Bray-Curtis, com os dados transformados por $\log (x+1)$ e a análise de agrupamento foi realizada pelo método da média aritmética ponderada (WPGMA) (Legendre \& Legendre, 1983), e representada na forma de um dendrograma. Para análise inversa (modo R), utilizou- se o índice de Bray-Curtis com os dados transformados por $\log (x+1)$. Em ambas as análises foram consideradas apenas as espécies constantes e acessórias, que totalizaram 23. Análises prévias realizadas com maior número de espécies evidenciaram os mesmos resultados obtidos com apenas as espécies constantes e acessórias.

\section{Resultados}

\section{Dados abióticos}

O tamanho do grāo do sedimento foi significativamente diferente entre os dois locais, embora estes não tenham diferido quanto ao grau de seleçāo (Tab. 1). No fundo sem vegetação, a areia foi classificada como areia grossa a muito grossa, moderada a pobremente selecionada. No banco de Halodule, o sedimento foi classificado como areia média a fina, pobremente ou muito pobremente selecionada.

A porcentagem de areia foi menor na área com Halodule do que na sem vegetação, enquanto que a fração silte-argila foi significativamente maior na primeira,

Tabela 1. Análise granulométrica: amplitude de variação do diâmetro médio do grão (Mz), desvio padrão, fração areia e silte-argila e conteúdo de matéria orgânica do sedimento da área com Halodule e da sem vegetação. Praia do Codó. $\mathrm{W}=$ teste de Wilcoxon. NS = diferenças não significantes $(p>0,05)$.

\section{Halodule Sem vegetação W Comparaç̄o}

\begin{tabular}{|c|c|c|c|c|}
\hline$N 2(\theta)$ & $1,0 \cdot 2,9$ & $-0,3 \cdot 0,9$ & 0 & $H>$ sV \\
\hline Desvio pactão & $1,6 \cdot 2,4$ & $0,6 \cdot 2,1$ & 12 & ws \\
\hline Areia $(x)$ & $78,4 \cdot 92,9$ & $85,7 \cdot 94,8$ & 1 & $H<\mathrm{sv}$ \\
\hline Silte-argila $(x)$ & $5,2 \cdot 18,0$ & $0,0 \cdot 8,3$ & 0 & $H>S V$ \\
\hline Matéria orgànica $(x)$ & $0,0 \cdot 1,7$ & $0,0-1,0$ & 5 & $H>S V$ \\
\hline
\end{tabular}

variando de 5,2 a $18 \%$ (Tab. 1). No local sem vegctaçāo, esta fração nāo foi observada em $60 \%$ das amostras. $O$ conteúdo orgânico variou de 0,1 a $1,7 \%$ no banco (Tab. 1), enquanto que na areia desprovida de vegetação não foi observado em $60 \%$ das amostras. Ambos os fatores foram maiores na presença de Halodule.

\section{Biomassa de Halodule}

Com relaçāo à biomassa das plantas de Halodule, observou-se que os valores mais altos ocorreram nos meses de dezembro de 1984 e março e dezembro de 1985 , enquanto que os mais baixos, no inverno e início da primavera (junho e setembro) (Fig. 3).

\section{Fauna}

O número de indivíduos das espécies da macrofauna bentônica por amostra $\left(78,5 \mathrm{~cm}^{2}\right)$ coletada na Praia do Cod6, em ambos os habitats, encontra-se no trabalho de Corbisier (1989).

Os poliquetos constituíram o grupo numericamente dominante nos dois locais (Tab. 2). Este grupo foi responsável por 69 a $82 \%$ do total da fauna no banco de Halodule, enquanto que no local sem vegetação, por 89 a 95\% da fauna total. No banco de Halodule, além de Polychaeta, Decapoda Anomura e Brachyura foram numericamente freqüentes representando $3 \mathrm{a} 8 \%$ do total da fauna, e Bivalvia, 3 a $7 \%$, além dos Oligochaeta (de 1 a $5 \%$ ) e Amphipoda (2 a 5\%). No local sem vegetação, Decapoda Anomura e Brachyura responderam por 0,5 a $3 \%$ do total, enquanto que Oligochaeta, 0,2 a $3 \%$, Gastropoda, 0 a 4\%, e Bivalvia, 0 a $2 \%$. Os Ophiuroidea ocorreram exclusivamente no banco de Halodule, enquanto que Bivalvia, Oligochaeta, Amphipoda, Decapoda Anomura e Brachyura, foram mais abundantes neste habitat (Tab. 2).

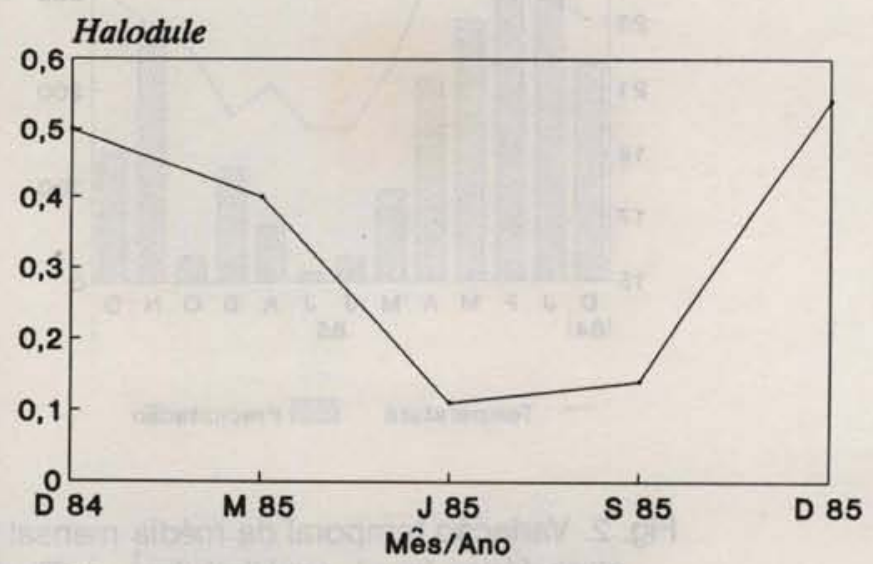

Fig. 3. Variaçäo temporal da média da biomassa de Halodule, em g peso seco por $78,5 \mathrm{~cm}^{-2}$. Praia do Codó, de dezembro de 1984 a dezembro de 1985. 
Tabela 2. Frequeência relativa dos grupos da macrofauna no total de amostras das áreas com Halodule e sem vegetação na Praia do Codó, em cada época de coleta

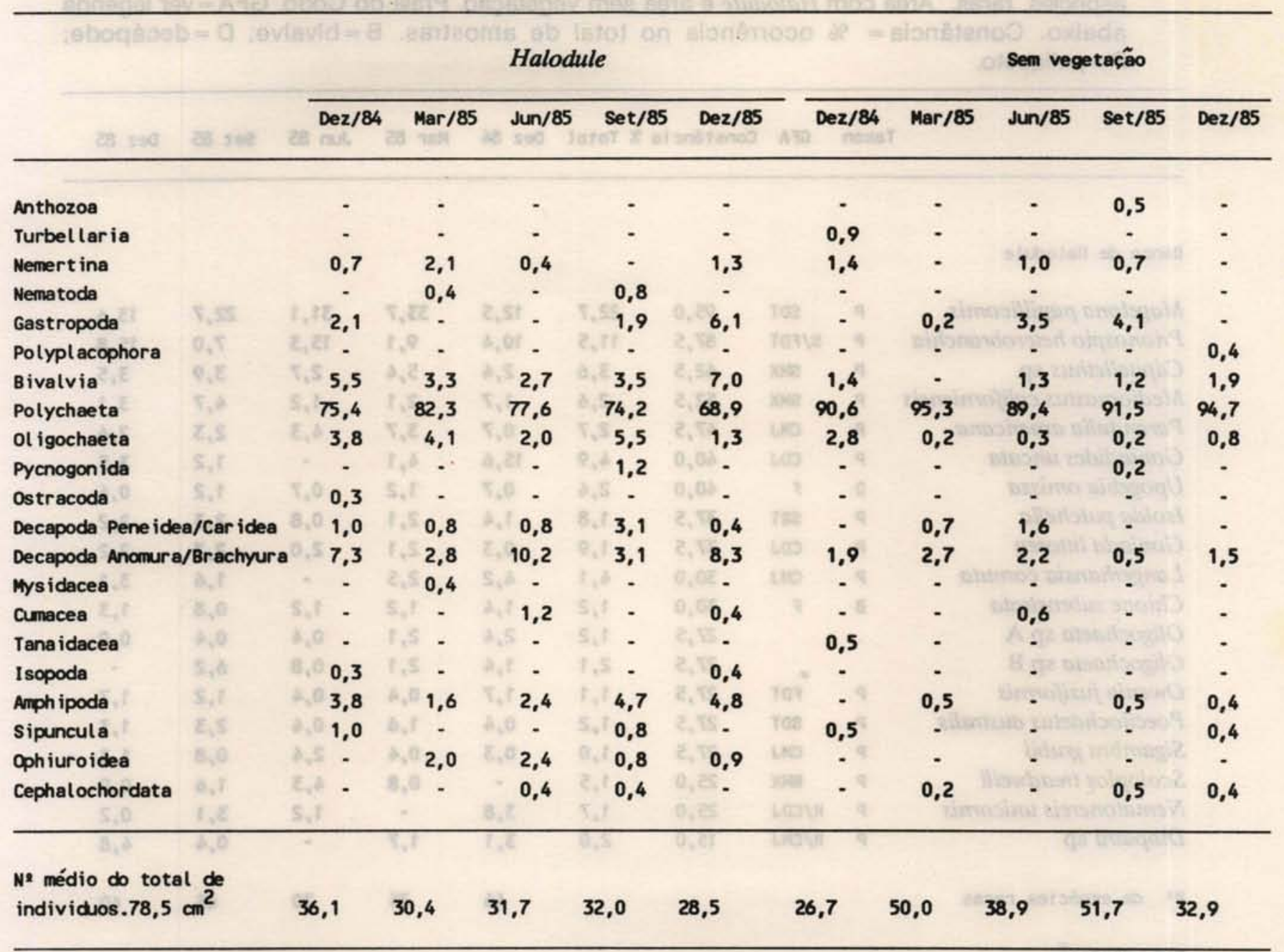

O poliqueto Magelona papillicomis foi a espécie mais abundante na área com Halodule (12,5 a 33,7\% do total), enquanto que Prionospio heterobranchia, a segunda ( 7,0 a $15,8 \%$ ) (Tab. 3). Capitellethus sp respondeu por 2,4 a 5,4\% do total, e Mediomastus califomiensis, 1,2 a $4,7 \%$. Estas espécies de poliquetos comedores de depósito de superfície foram as quatro espécies constantes neste habitat, por todo o período do estudo. Treze espécies de poliquetos de diversos grupos tróficos constituiram as espécies acessórias, assim como os filtradores Chione subrostrata (bivalve) e o anomura Upogebia omissa, além de dois oligoquetos (Tab. 3).

No local desprovido de vegetação, seis espécies de poliquetos foram constantes: Exogone arenosa respondeu por 24,4 a 44,2\% do total da fauna; Goniadides uncata, por 11,8 a $32,3 \%$, Prionospio heterobranchia, por 7,9 a $18,3 \%$, Aricidea fauveli, por 6,0 a $14,3 \%$, Nematonereis unicomis por 0,8 a $6,5 \%$ e Scolelepis squamata por 1,2 a $4,7 \%$ (Tab. 3). Desses, três poliquetos são espécies carnívoras/omnívoras e os dois espionídeos, $P$. heterobranchia e $S$. squamata, são comedores de depósito ou suspensívoros.

A análise de agrupamento mostrou que os dois locais diferiram em relaçāo à composiçāo espećfica durante todo o período do estudo, a nível de mais de $50 \%$ de dissimilaridade (Fig. 4). A análise inversa distinguiu dois grupos principais de espécies (Fig. 5). O grupo A, associado à presença de Halodule, foi constituído por dezesseis espécies que são, basicamente, as três constantes, exceto $P$. heterobranchia, e as acessórias da área com Halodule (Tab. 3); e o grupo B, relacionado à área sem a vegetação, constituído pelas seis espécies constantes e mais a acessória Linophenus ambigua, deste local (Tab. 3).

$\mathrm{Na}$ Tabela 4, encontra-se a comparaçāo das características estruturais das associaçōes macrofaunísticas dos dois locais e entre os diferentes períodos de coleta. O número total de indivíduos por 
Tabela 3. Freqüência relativa (\%) das espécies constantes e acessorlas no total de indivíduos, do total e de cada coleta, e seus respectivos grupos funcionais de alimentação (GFA). Número de espécies raras. Área com Halodule e área sem vegetação. Praia do Codó. GFA=ver legenda abaixo. Constância $=\%$ ocorrência no total de amostras. $B=$ bivalve; $D=$ decápode; $\mathrm{P}=$ poliqueto.

Banco de Halodule

\begin{tabular}{|c|c|c|c|c|c|c|c|c|c|}
\hline Magelona papillicomis & $\mathbf{P}$ & SDT & 95,0 & 22,7 & 12,5 & 33,7 & 31,1 & 22,7 & 13,6 \\
\hline Prionospio heterobranchia & $\mathbf{P}$ & S/FDT & 87,5 & 11,5 & 10,4 & 9,1 & 15,3 & 7,0 & 15,8 \\
\hline Capitellethus sp & $\mathbf{P}$ & $\operatorname{sen}$ & 62,5 & 3,6 & 2,4 & 5,4 & 2,7 & 3,9 & 3,5 \\
\hline Mediomastus californiensis & $\mathbf{P}$ & $\sin x$ & 52,5 & 2,6 & 1,7 & 2,1 & 1,2 & 4,7 & 3,1 \\
\hline Parandalia americana & $\mathbf{P}$ & CMs & 47,5 & 2,7 & 0,7 & 3,7 & 4,3 & 2,3 & 2,6 \\
\hline Goniadides uncata & $\mathbf{P}$ & CDJ & 40,0 & 4,9 & 15,6 & 4,1 & - & 1,2 & 3,5 \\
\hline Upogebia omissa & D & $\mathbf{F}$ & 40,0 & 2,4 & 0,7 & 1,2 & 0,7 & 1,2 & 0,4 \\
\hline Isolda pulchella & $\mathbf{P}$ & SST & 37,5 & 1,8 & 1,4 & 2,1 & 0,8 & 2,3 & 2,2 \\
\hline Goniada littorea & $\mathbf{P}$ & CDJ & 37,5 & 1,9 & 0,3 & 2,1 & 2,0 & 2,7 & 2,2 \\
\hline Langerhansia comuta & $\mathbf{P}$ & CMs & 30,0 & 4,1 & 4,2 & 2,5 & - & 1,6 & 3,1 \\
\hline Chione subrostrata & B & $\mathbf{F}$ & 30,0 & 1,2 & 1,4 & 1,2 & 1,2 & 0,8 & 1,3 \\
\hline Oligochaeta sp A & $z$ & 0 & 27,5 & 1,2 & 2,4 & 2,1 & 0,4 & 0,4 & 0,9 \\
\hline Oligochaeta sp B & & & 27,5 & 2,1 & 1,4 & 2,1 & 0,8 & 6,2 & - \\
\hline Owenia fusiformis & $\mathbf{P}$ & FDT & 27,5 & 1,1 & 1,7 & 0,4 & 0,4 & 1,2 & 1,7 \\
\hline Poecilochaetus australis & $\mathbf{P}$ & SDT & 27,5 & 1,2 & 0,4 & 1,6 & 0,4 & 2,3 & 1,3 \\
\hline Sigambra grubii & $\mathbf{P}$ & CMs & 27,5 & 1,0 & 0,3 & 0,4 & 2,4 & 0,8 & 1,3 \\
\hline Scoloplos treadwelli & $\mathbf{P}$ & $\operatorname{Ban} x$ & 25,0 & 1,5 & $=0$ & 0,8 & 4,3 & 1,6 & 0,9 \\
\hline Nematonereis unicornis & $\mathbf{P}$ & H/CDJ & 25,0 & 1,7 & 3,8 & - & 1,2 & 3,1 & 0,2 \\
\hline Diopatra sp & $\mathbf{P}$ & H/CMJ & 15,0 & 2,0 & 3,1 & 1,7 & $\cdot$ & 0,4 & 4,8 \\
\hline N2 de espécies raras & & 15, 25 & 4,85 & 0,8 & 46 & 35 & 30 & 45 & 40 \\
\hline \multicolumn{10}{|l|}{ Sem vegetação } \\
\hline Exogone arenosa & $\mathbf{P}$ & HMJ & 85,0 & 30,1 & 27,1 & 44,2 & 24,4 & 23,7 & 31,2 \\
\hline Goniadides uncata & $\mathbf{P}$ & CDJ & 95,0 & 20,1 & 27,1 & 11,8 & 13,2 & 16,2 & 32,3 \\
\hline Prionospio heterobranchia & $\mathbf{P}$ & S/FDT & 92,5 & 12,5 & 7,9 & 13,1 & 18,3 & 16,9 & 6,5 \\
\hline Aricidea fauveli & $\mathbf{P}$ & SHX & 57,5 & 11,9 & 11,2 & 14,3 & 16,4 & 6,0 & 11,4 \\
\hline Nematonereis unicornis & P & $\mathrm{H} / \mathrm{CDJ}$ & 62,5 & 4,2 & 3,7 & 3,7 & 6,4 & 6,5 & 0,8 \\
\hline Scolelepis squamata & $\mathbf{P}$ & S/FDT & 57,5 & 3,0 & 4,7 & 1,2 & 2,6 & 3,1 & 3,4 \\
\hline Linophenus ambigua & P & $\cos x$ & 45,0 & 2,6 & 3,3 & 1,5 & 2,2 & 5,3 & 0,8 \\
\hline Mediomastus californiensis & P & $\operatorname{sen} x$ & 30,0 & 1,0 & 0,9 & 0,5 & 1,3 & 2,2 & $\cdot$ \\
\hline W' de espécies raras & & +528 & & & & 23 & 23 & 29 & 22 \\
\hline
\end{tabular}

Legendas para GFA:

Letras maiúsculas

na primeira posição:

B - comedor de detrito de subsuperfície

C - carnivoro

F - filtrador

$H$ - herbivoro

S - comedor de detrito de superfície na segunda posição:

D - discretamente móvel

M - móvel

S - séssil na terceira posição:

$\mathrm{J}$ - com mandibulas

P - com mecanismo de sucção

$T$ - com tentáculos

$X$ - com outras estruturas, em geral, faringes inermes eversíve is 


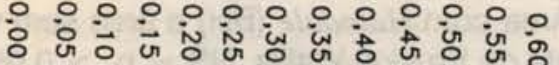

BH1 MAR 85

BH2 DEZ 85

BH1 SET 85

BH2 SET 85

BH2 MAR 85

BH1 JUN 85

BH2 JUN 85

BH2 DEZ 84

BH1 DEZ 85

BH1 DEZ 84

SV1 DEZ 84

SV1 DEZ 85

SV1 MAR 85

SV1 JUN 85

SV2 MAR 85

SV2 JUN 85

SV1 SET 85

SV2 SET 85

SV2 DEZ 84

SV2 DEZ 85

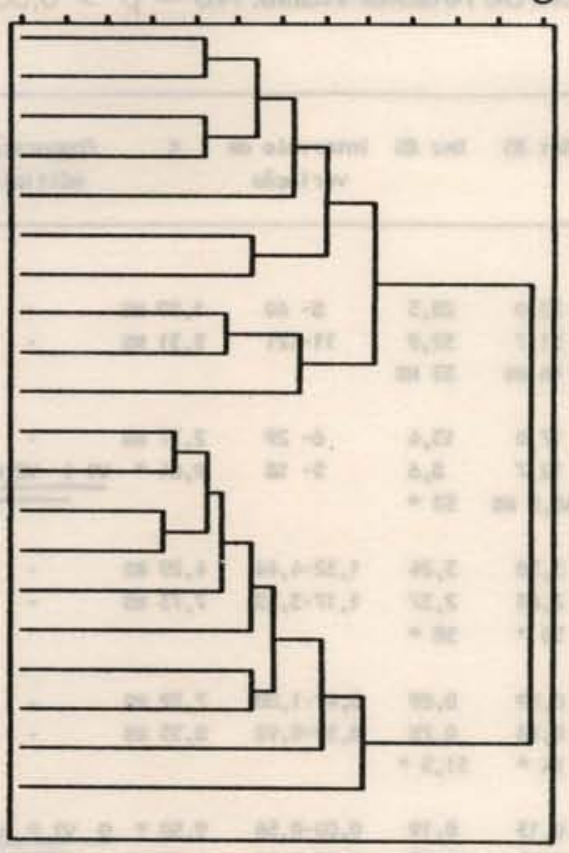

Fig. 4. Dendograma da análise de agrupamento das amostras sazonais da área com Halodule $(\mathrm{BH})$ e da sem vegetação (SV), em 1984/1985. Indice de dissimilaridade de Bray-Curtis e agrupamento por WPGMA. amostra variou de 8 a 60 no banco de Halodule, e de 11 a 121 na área sem vegetação. Estes números não foram significativamente diferentes; contudo, o intervalo de variaçāo dos valores foi maior no local sem vegetaçāo. $O$ número de espécies variou de 6 a 29 por amostra no banco de Halodule, e de 5 a 18 no outro local, sendo em geral menor neste. $O$ índice de diversidade variou de 1,32 a 4,44 no banco, e de 1,17 a 3,53 no outro local. Este índice foi maior na presença de Halodule, em todas as coletas. A eqüitatividade foi menor no local sem vegetação apenas em duas ocasiōes e variou de 0,39 a 0,92 , e na área com Halodule variou de 0,47 a 1,00.

A relação da epifauna com a fauna total foi maior no banco de Halodule (Tab. 4). Inversamente, os poliquetos com mobilidade em relação ao total de poliquetos foram mais abundantes na área desprovida de vegetaçāo.

Verifica-se, na Figura 6, a tendência de variação do número médio do total de indivíduos e de espécies, no período do estudo, bem como da média dos índices de diversidade e equitatividade nos dois locais. Em relaçāo ao número total de indivíduos, a análise de variância não indicou diferenças significantes entre os perfodos do ano, nos dois locais (Tab. 4); no entanto, a variaçāo do número de indivíduos foi mais ampla no fundo sem vegetação. De um modo geral, os maiores números de espécies e diversidade foram observados em setembro (primavera), embora as diferenças não sejam significativas (Tab. 4). A eqüitatividade não apresentou diferenças significativas no período do estudo, nos dois locais. Quanto às relaçōes
Fig. 5. Dendograma da análise de agrupamento das espécies constantes e acessórias da área com Halodule e da sem vegetação, em 1984/1985. Indice de dissimilaridade de Bray-Curtis e agrupamento por WPGMA.

Goniada littorea
Paradalia americana
Capitellethus sp
Isolda pulchella
Poecilocahetus australis
Oligochaeta sp B
Mediomastus califomiensis
Upogebia amissa
Scoloplos treadwelli
Sigambra grubii
Chione subrostrata
Oligochaeta sp A
Langerhansia comuta
Owenia fusiformis
Diopatra sp
Magelona papillicomis
Exogone arenosa
Goniadides uncata
Prionospio heterobanchia
Aricidea fauveli
Linophenus ambigua
Nematonereis unicomis
Scolelepis squamata

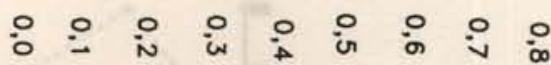

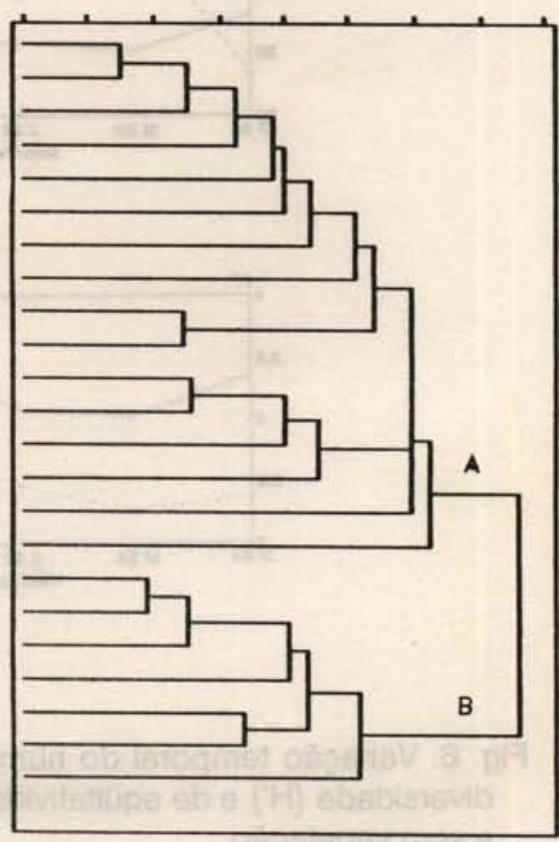


Tabela 4. Média por coleta e intervalo de variação, no período do estudo, do número de indivíduos e de espécies, dos indices de diversidade e eqüitatividade, das relaçōes epifauna/fauna total e poliquetos errantes/total de poliquetos por amostra $\left(78,5 \mathrm{~cm}^{2}\right)$. Área com Halodule $(\mathrm{BH})$ e área sem vegetação (SV). Praia do Codó. MW = teste de Mann-Whitney. $K=$ teste de Kruskal-Wallis. NS $=p>0,05 ;\left(^{*}\right) p<0,05$

Dez 84 Mar 85 Jun 85 Set 85 Dez 85 Intervalo de $K$ Corparacões varieç̃o múttiplas

\begin{tabular}{|c|c|c|c|c|c|c|c|c|c|}
\hline \multicolumn{10}{|c|}{ Minero de individuos } \\
\hline BH & 36,1 & 30,4 & 31,7 & 32,0 & 28,5 & 8. 60 & 1,59 ws & - & , \\
\hline sv & 26,7 & 50,0 & 38,9 & 51,7 & 32,9 & $11-121$ & 5,31 ws & - & \\
\hline $\mathbf{m}$ & 42 NS & 39 wS & 42,5 wS & 48 ws & 33 us & & & & \\
\hline \multicolumn{10}{|c|}{ Winero de esptcies } \\
\hline BH & 16,9 & 13,0 & 12,6 & 17,0 & 13,4 & 6- 29 & $2, \pi$ ws & $\cdot$ & \\
\hline sv & 8,4 & 9,0 & 8,6 & 12,7 & 8,6 & 5- 18 & $9,84=$ & $\begin{array}{lll}V_{1} & \mathrm{~V} \\
\end{array}$ & o P \\
\hline $\mathbf{M}$ & $51 *$ & 48,5 ws & $59,5 *$ & 46,5 wS & $53 \cdot$ & & & & \\
\hline \multicolumn{10}{|c|}{ Divers idade } \\
\hline вн & 3,36 & 3,03 & 3,06 & 3,58 & 3,24 & $1,32-4,44$ & $4,29 \mathrm{MS}$ & $\cdot$ & \\
\hline sv & 2,37 & 2,23 & 2,60 & 2,83 & 2,37 & $1,17-3,53$ & 7,73 us & $\cdot$ & \\
\hline \multirow{2}{*}{\multicolumn{10}{|c|}{ Equitatividade }} \\
\hline & & & & & & & & & \\
\hline BH & 0,85 & 0,82 & 0,84 & 0,89 & 0,89 & $0,47-1,00$ & $\begin{array}{l}7,22 \mathrm{ws} \\
0,35 \mathrm{us}\end{array}$ & - & \\
\hline sv & $\begin{array}{l}0,80 \\
50 \text { ws }\end{array}$ & $\begin{array}{l}0,73 \\
35,5 \text { ws }\end{array}$ & $\begin{array}{c}0,80 \\
41,5 \text { Ns }\end{array}$ & 0,78 & $\begin{array}{c}0,78 \\
51,5\end{array}$ & $0,39-0,92$ & 0,35 wS & & \\
\hline \multicolumn{10}{|c|}{ Epifauna/fana total } \\
\hline BM & 0,10 & 0,05 & 0,16 & 0,13 & 0,19 & $0,00-0,56$ & $9,50 \cdots$ & O V1 P & $\sqrt{2} I$ \\
\hline sv & 0,03 & 0,06 & 0,09 & 0,04 & 0,03 & $0,00-0,25$ & 4,22 ws & 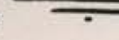 & \\
\hline $\mathbf{m}$ & $51 *$ & 34,5 wS & 48,5 us & $54 *$ & $55 *$ & & & & \\
\hline \multicolumn{10}{|c|}{ Pol iqueto errante/total de pol iqueto } \\
\hline BH & 0,47 & 0,26 & 0,17 & 0,33 & 0,41 & $0,07-1,00$ & $15,48 *$ & $10 P$ & V2 V1 \\
\hline sv & 0,71 & 0,55 & 0,58 & 0,56 & 0,74 & $0,33-0,90$ & 5,96 ws & $=$ & \\
\hline $\mathbf{m}$ & 49 wS & 57 & $63 \cdot$ & $59 \cdot$ & $56 \cdot$ & 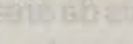 & Koxyet & afine & 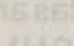 \\
\hline
\end{tabular}
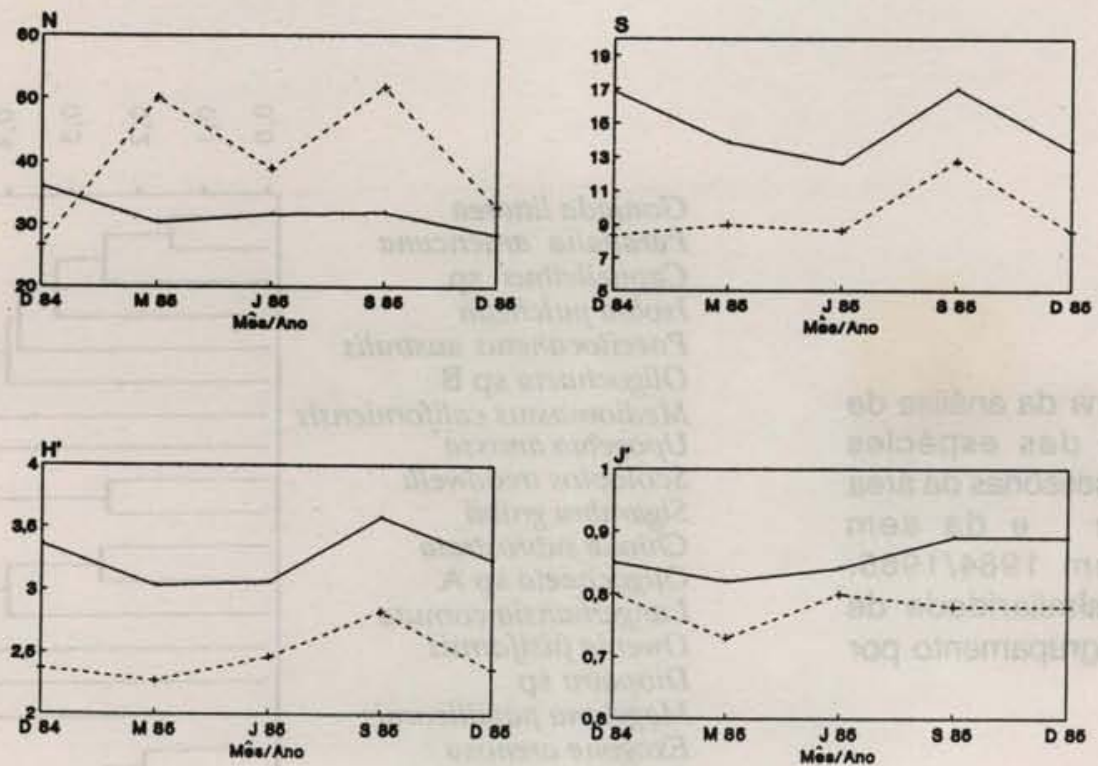

- Halodule ...+... Sem vegetacảo

Fig. 6. Variação temporal do número médio de individuos $(\mathrm{N})$ e de espécies (S), da média do índice de diversidade $\left(\mathrm{H}^{\prime}\right)$ e de eqüitatividade $\left(\mathrm{J}^{\prime}\right)$ da macrofauna bentônica por $78,5 \mathrm{~cm}^{2}$, nas áreas com Halodule e sem vegetação; 

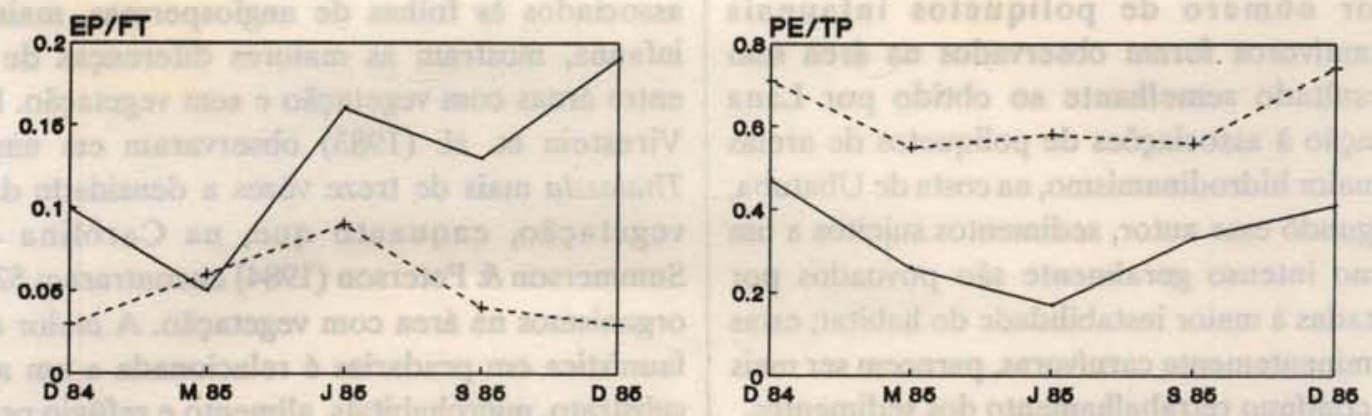

—Halodule ‥+- Sem vegetação

Fig. 7. Variação temporal da média da relação epifauna/fauna total (EP/FT) e de poliquetos errantes/total de poliquetos (PE/TP) da macrofauna bentônica por $78,5 \mathrm{~cm}^{2}$, nas áreas com Halodule e sem vegetação;

epifauna/fauna total e poliquetos com mobilidade/total de poliquetos, essas mostraram alguma variação temporal na área com Halodule, sendo que os poliquetos com mobilidade foram mais abundantes no verão, enquanto que a epifauna foi mais abundante no inverno (Fig. 7, Tabela 4). Com relação à composição específica, esta manteve-se estável durante todo o período do estudo nos dois locais, nāo se observando modificação notável das espécies constantes e acessórias (Tab. 3), o que se refletiu nos agrupamentos das amostras (Fig. 4).

\section{Discussão}

$\mathrm{Na}$ franja do infralitoral da Praia do Codo, Ubatuba, São Paulo, ocorrem duas associaçōes de espécies do macrobentos distintas. Estas associaçōes ainda não haviam sido relatadas naquela praia, uma vez que Amaral (1979, 1980) estudou os poliquetos da zona entremarés e do infralitoral abaixo de $1 \mathrm{~m}$ de profundidade. Esta autora comentou o fato da alta diversidade de espécies de poliquetos na regiāo inferior da praia.

As duas associaçōes estão relacionadas à presença de Halodule wrightii ou ao sedimento sem vegetação. Este fato foi observado em outras localidades, com presença de angiospermas (por exemplo, Santos \& Simon, 1974; Orth, 1977; Reise, 1977; Stoner, 1980; Lewis \& Stoner, 1983; Summerson \& Peterson, 1984; Lana \& Guiss, 1991). As associaçōes sẩo numericamente dominadas por espécies diferentes de poliquetos infaunais, exceto por Prionospio heterobranchia. No banco, predominaram poliquetos infaunais comedores de depósitos de superfície e, no local sem vegetaçāo, formas carnívoras/omnívoras, com estruturas bucais mais desenvolvidas (mandíbulas) e alguns suspensívoros. Com relação a $P$. heterobranchia, esta espécie pertence à família Spionidae caracterizada como "bitrofica", comedora de depósito de superfície/suspensívora, que varia a forma de alimentação dependendo das condiçōes hidrográficas do fundo (Dauer et al., 1981; Maurer \& Leathem, 1981).

O tamanho do grão do sedimento é um fator importante que determina a ocorrência de associaçōes de espécies em fundos moles (Gray, 1974) e a infauna, em bancos de angiospermas marinhas (Virnstein, 1987). No entanto, como Stoner (1980) observou, locais com diferentes graus de biomassa de macrófitas são geralmente acompanhados de variaçōes granulométricas no sedimento, fato que dificulta a interpretação dos resultados em relação à influência dos fatores tipo de sedimento e presença de plantas sobre a composição da infauna. Além disso, é reconhecida a capacidade das angiospermas marinhas de reduzir correntes e alterar o ambiente sedimentar, podendo, desse modo, afetar indiretamente a composição da infauna, como Orth et al. (1984) apontaram.

No banco de Halodule formas infaunais comedoras de depósitos de superfície dominaram. Outros autores têm salientado a predominância de inverterbrados comedores de depósito em bancos de angiospermas marinhas (Young \& Young, 1978; Lewis \& Stoner, 1983). Ao contrário, Stoner (1980) verificou um decréscimo no número de poliquetos comedores de depósito com o aumento da biomassa da vegetação, enquanto que poliquetos suspensívoros e carnívoros aumentaram em abundância. $\mathrm{Na}$ Praia do Cod6, a maior estabilidade no fundo com vegetação permite, além daquela predominância, a presença do anomura Upogebia omissa, que vive em galerias, e do poliqueto tubícola Isolda pulchella. A presença deste último vem sendo relacionada aos rizomas de angiospermas, como Spartina altemiflora (Takeda, 1988; Lana \& Guiss,1991). 
Um maior número de poliquetos infaunais predadores-omnfvoros foram observados na área sem vegetação, resultado semelhante ao obtido por Lana (1981) em relação à associações de poliquetos de areias submetidas a maior hidrodinamismo, na costa de Ubatuba, São Paulo. Segundo esse autor, sedimentos sujeitos a um hidrodinamismo intenso geralmente são povoados por espécies adaptadas à maior instabilidade do habitat; estas formas, predominantemente carnívoras, parecem ser mais resistentes ao contínuo retrabalhamento dos sedimentos.

Desse modo, em função das diferenças observadas no tipo de sedimento e sua estabilidade e, secundariamente, em função da presença das plantas as diferenças na composição da macrofauna podem ser explicadas.

A expressiva dominância de poliquetos infaunais, na área com Halodule, difere em parte dos resultados de trabalhos realizados em pradarias de angiospermas marinhas nos E.U.A., nos quais também foi utilizado o mesmo tipo de amostrador para coletar os invertebrados (resumidos em Virnstein, 1987). Na maioria desses estudos, poliquetos dividem a dominância numérica com gastrópodes e crustáceos peracáridos epifaunais. Uma das razōes para explicar aquele fato seria o pequeno tamanho das plantas de Halodule, quando comparada a outras espécies de angiospermas, além da ocorrência de poucas macroalgas epífitas, o que propiciaria menos substrato e microhabitats, além de menos refúgio contra a predação, para espécies epifaunais.

Apesar deste fato, o maior numero de organismos epifaunais, tais como ofiuroides, anfipodes e decápodes, encontrados na área com Halodule deve estar diretamente relacionado à presença das folhas, que, embora não muito desenvolvidas, ainda assim provêm algum substrato para espécies epifaunais, além de alimento e heterogeneidade espacial (Lewis, 1984; Orth et al., 1984; Summerson \& Peterson, 1984; Wells et al., 1985; Edgar, 1990a; Schneider \& Mann, 1991).

No banco de Halodule também foi observada uma maior diversidade de espécies, que refletiu um maior número de espécies, e não uma maior eqüitatividade; tal fato é reflexo dos fatores acima mencionados relacionados à presença da vegetação. Diversos trabalhos relativos à fauna de bancos de angiospermas marinhas e áreas desprovidas de vegetaçāo registram o fato da ocorrência de maior diversidade de espécies nos primeiros (Lewis, 1984; Orth et al., 1984; Summerson \& Peterson, 1984; Edgar, 1990a; Lana \& Guiss, 1991).

Entretanto, não foi observada diferença significativa da densidade faunística entre o banco de Halodule e a área próxima sem vegetação, fato relatado para outras localidades (Santos e Simon, 1974; Orth et al., 1984; Summerson e Peterson, 1984; Wells et al., 1985; Edgar, 1990a; Lana \& Guiss, 1991). Animais epifaunais associados às folhas de angiospermas, mais do que a infauna, mostram as maiores diferenças de densidade entre áreas com vegetação e sem vegetação. Na Flórida, Virnstein et. al. (1983) observaram em um banco de Thalassia mais de treze vezes a densidade da área sem vegetação, enquanto que, na Carolina do Norte, Summerson \& Peterson (1984) encontraram 52 vezes mais organismos na área com vegetação. A maior abundância faunística em pradarias é relacionada a um aumento de substrato, microhabitats, alimento e refugio providos pela vegetação. $O$ fato observado na Praia do Codó poderia ser explicado, em parte, pelo tamanho pequeno da angiosperma.

Um resultado semelhante ao observado na Praia do Cod6 foi obtido por Young \& Young (1982) na laguna de Carrie Bow, Belize, e explicado pela intensa pressão de predaçāo na área com vegetaçāo estudada. Entre as hipoteses que poderiam explicar este fato, uma seria a de que a predação por pequenos caranguejos e camarōes, geralmente relacionados às plantas, mantém a densidade da fauna bentônica a níveis mais baixos, como foi observado por Nelson (1981a,b) e Leber (1985); no entanto, as densidades daqueles crustáceos nāo foram muito altas nas amostras coletadas na Praia do Cod6. Uma segunda razão seria a de que o pequeno tamanho das plantas de Halodule nāo propiciaria refúgio à macrofauna contra os predadores epibentônicos, fato que foi verificado através do uso de experimentos com gaiolas de exclusāo na Praia do Cod6 (Corbisier, 1989); desse modo, a predaçāo pode manter a densidade faunística na área do banco abaixo da capacidade suporte do ambiente.

Em geral, comunidades de fundos sem vegetação parecem estar sujeitas à uma maior pressão de predação que em fundos com vegetação (Summerson \& Peterson, 1984). Os experimentos com gaiolas de exclusāo realizados na Praia do Codó não levaram a esta conclusāo (Corbisier, 1989). Os resultados aqui analisados sugerem que a instabilidade do sedimento $e$ o fator principal responsável pelo observado no local desprovido de vegetação. Esta instabilidade refletiu-se na composiçāo especifica deste local, bem como na maior amplitude de variação das densidades, sugerindo uma distribuição em mosaico da fauna mais evidente; este fato, segundo Thistle (1981), ocorre em áreas sujeitas a perturbaçōes físicas, devido às diferentes estratégias de colonização que as espécies possuem para ocupar espaços recém abertos.

Não se verificou um padrão de variação sazonal da estrutura da comunidade muito pronunciado $e$ as variaçōes observadas não foram semelhantes nos dois locais, assim como não acompanharam as flutuaçōes da temperatura e salinidade da água. $\mathrm{Na}$ areia desprovida de vegetaçāo, as amplitudes de variação da densidade faunística foram maiores entre amostras do que entre períodos do ano devido, provavelmente, à maior 
instabilidade do sedimento, como mencionado anteriormente. $\mathrm{Na}$ área de Halodule, as variaçōes foram muito pequenas e não houve distinção nítida na composição específica entre as amostras de verāo, primavera ou outono, principalmente.

Segundo Virnstein (1987), presume-se que variaçōes sazonais de temperatura e luz solar sejam a causa principal de padrōes sazonais em comunidades de angiospermas marinhas. Nos trabalhos que analisaram a variação sazonal da abundância da fauna (epi e/ou infauna) de pradarias desse tipo de vegetação, atribuiu-se essa variação à variação da biomassa das angiospermas (Stoner, 1983), ou ao aumento de macroalgas epifíticas (Lewis, 1987), de alimento (microalgas) (Edgar, 1990b), ou, ainda, de macrófitas à deriva (Edgar, 1990a) e, principalmente, à variação da abundância de predadores, como peixes e/ou decápodes predadores (Santos \& Simon, 1974; Stoner, 1980; Nelson et al., 1982; Sheridan \& Livingston, 1983).

$\mathrm{Na}$ Praia do Codo, a variação da biomassa de Halodule acompanhou a variação da temperatura (Figs 2 e 3 ). No entanto, nāo houve, aparentemente, conseqüente variaçāo da abundância da fauna. Como os outros fatores, que poderiam ser a causa da variabilidade da fauna, não foram aqui analisados, nâo se pode discutí-los; entretanto, a exclusão experimental de macropredadores, nos mesmos períodos (Corbisier, 1989), indicou que a redução ou o não aumento da abundância da macrofauna coincidiu com o maior efeito da predação sobre as associações faunística na área com Halodule.

\section{Agradecimentos}

Este trabalho é parte da tese de doutorado da autora, que expressa seu agradecimento ao Prof. Tit. Luiz R. Tommasi pela orientação. Este trabalho recebeu auxílio à pesquisa da FAPESP (proc.no. 84/2812-8) e a autora, bolsa da FAPESP (proc.no. 84/1091-5) e da CAPES. A identificaçāo dos diversos grupos faunísticos foi realizada pelos Professores Ana Maria S. Pires, Franci M. Fancinato Varoli, Fosca P. P. Leite, Gustavo A. Schmidt de Melo, Luiz R. Tommasi, Montanaga Iwai, Sônia G. B. C. Lopes, Adolpho Birman, Sérgio Didati, e a M.Sc. M. Fernanda L. dos Santos. Os Professores A. Cecilia Z. Amaral, Edmundo F. Nonato e Paulo da C. Lana auxiliaram na identificação dos Polychaeta. A todos o nosso agradecimento.

\section{Referências bibliográficas}

AMARAL, A. C. Z. 1979. Ecologia e contribuição dos anelídeos poliquetos para a biomassa bêntica da zona entre-marés, no litoral norte do Estado de São Paulo. Bolm Inst. oceanogr., S Paulo, 28:1-52.
AMARAL, A. C. Z. 1980. Anelídeos poliquetos do infralitoral em duas enseadas da região de Ubatuba. II. Aspectos ecológicos. Bolm Inst. oceanogr., S Paulo, 29:69-87.

ASMUS, M. L. 1984. Estrutura da comunidade associada a Ruppia maritima no estuário da Lagoa dos Patos, Rio Grande do Sul, Brasil. Dissertaçāo de mestrado. Universidade do Rio Grande, 154 p.

BELL, J. D. \& WESTOBY, M. 1986. Abundance of macrofauna in dense seagrass is due to habitat preference, not predation. Oecologia (Berlin), 68:205-209.

BOUSFIELD, E. L. 1973. Shallow water gammaridean amphipods of New England. Ithaca, Cornell University Press, 312 p.

CORBISIER, T. N. 1989. Aspectos ecologicos das associaçōes de espécies da macrofauna bentônica e avaliaçāo do efeito da predaçāo sobre a sua estrutura na Praia do Codó (Ubatuba, SP, Brasil). Tese de doutorado. Universidade de São Paulo, Instituto Oceanográfico, 144 p.

; ESTON, V. R. de; OLIVEIRA Filho, E. C. de \& Ambrósio JUNIOR, O. 1986. Ecologia de um banco de angiospermas marinhas do litoral do Estado de São Paulo. Mini-Simpósio de Biologia Marinha, 5., São Sebastiāo, 1986. Resumos. CEBIMar-USP, p. 6.

DAJOZ, R. 1973. Ecologia Geral. Sāo Paulo, Vozes/EDUSP, 472 p.

DAUER, D.M.; MAYBURY, C.A. \& EWING, R.M. 1981. Feeding bahavior and general ecology of several spionid polychaetes from Chesapeake Bay. J. expl mar. Biol. Ecol., 54:21-38.

EDGAR, G. J. 1990a . The influence of plant structure on the species richness, biomass and secondary production of macrofaunal assemblages associated with Western Australian seagrass beds. J. expl mar. Biol. Ecol., 137: 215-240.

1990b. Population regulation, population dynamics and competition amongst mobile epifauna associated with seagrass. J. expl mar. Biol. Ecol., 144:205-234.

FAUCHALD, K. \& JUMARS, P. A. 1979. The diet of worms: a study of polychaete-feeding guilds. Oceanogr. mar. Biol. a. Rev.,17:193-284. 
FREDETTE, T.J.; DIAZ, R. J.; VAN MONTFRANS, J. \& ORTH, R. J. 1990. Secondary production within a seagrass bed (Zostera marina and Ruppia maritima) in lower Chesapeake Bay. Estuaries, 13:431-440.

GIBBONS, J. D. 1976. Nonparametric methods for quantitative analysis. Holt, Rinehart and Wiston, $463 \mathrm{p}$.

GRAY, J. S. 1974. Animal-sediment relationships. Oceanogr. mar. Biol. a. Rev.,12:223-261.

GROSS, M. G. 1971. Carbon determination. In: Carved, R. E., ed. Procedures in sedimentary petrology. New York, Wiley-Interscience, p. 573-596.

HECK JR, K. L. \& WETSTONE, G. S. 1977. Habitat complexity and species richness and abundance in tropical seagrass meadows. J. Biogeog., 4:135-142.

LANA, P. da C. 1981. Padrōes de distribuiçāo e diversidade específica de anelídeos poliquetos na regiāo de Ubatuba, Estado de São Paulo. Dissertação de mestrado. Universidade de São Paulo, Instituto Oceanográfico, 111 p.

\& GUISS, C. 1991. Influence of Spartina alterniflora on stucture and temporal variability of macrobenthic associations in a tidal flat of Paranaguá Bay (sotheastern Brazil). Mar. Ecol. Prog. Ser., 73:231-244.

LEBER, K. M. 1985. The influence of predatory decapods, refuge, and microhabitats selection on seagrass communities. Ecology, 66:1951-1964.

LEGENDRE, L. \& LEGENDRE, P. 1983. Numerical ecology. Amsterdam, Elsevier, 419 p.

LEWIS, F. G. 1984. Distribution of macrobenthic crustaceans associated with Thalassia, Halodule and bare sand substrata. Mar. Ecol. Prog. Ser., 19:101-113.

1987. Crustacean epifauna of seagrass and macroalgae in Apalachee Bay, Florida, USA. Mar. Biol., 94:219- 229.

\& Stoner, A. W. 1983. Distribution of macrofauna within seagrass beds: an explanation for patterns of abundance. Bull. mar. Sci. 33:296-304.

LOPES, S. G. B. C. 1983. Levantamento das espécies de Gastropoda e Bivalvia do Saco da Ribeira e taxocenose dos Bivalvia da Praia do Saco da Ribeira, Ubatuba, SP. Dissertação de mestrado. Universidade de São Paulo, Instituto Oceanográfico, 161 p.
MAURER, D. \& LEATHEM, W. 1981. Polychacte feeding guilds from George Bank, USA. Mar. Biol., 62:161-171.

MCROY, C. P. \& HELFFERICH, C. 1977. Seagrass ecosystems: a scientific perspective. New York, Marcel Dekker, 314 p.

MESQUITA, A. R. DE \& HARARI, J. 1983. Tides and tide gauges of Canananeia and Ubatuba-Brazil (lat $24^{\circ}$ ). Relat. int. Inst. Oceanogr. Univ. S Paulo, 11:1-14.

NELSON, W. G. 1981a. The role of predation by decapod crustaceans in seagrass ecosystems. Kieler Meeresforsch., Sonderh., 5:529- 536.

1981b. Experimental studies of decapod and fish predation on seagrass macrobenthos. Mar. Ecol. Prog. Ser., 5:141-149

; Cairns, K. C. \& Virnstein, R. W. 1982. Seasonality and spatial patterns of seagrass-associated amphipods of the Indian River lagoon, Florida. Bull. mar. Sci., 32:121-129.

OLIVEIRA FILHO, E. C. de; PIRANI, J. R. \& GIULETTI, A. M. 1983. The Brazilian seagrasses. Aquat. Bot., 16:251-267.

ORTH, R.J. 1977. The importance of sediment stability in seagrass communities. In: Coull, B. C., ed. Ecology of marine benthos. Columbia, Univ. South Carolina Press, p. 281-300

, HECK, K. L. \& VAN MONTFRANS, J. 1984. Faunal communities in seagrass beds: a review of the influence of plant structure and prey characteristics on predator-prey relationships. Estuaries, 7:339-350.

REISE, K. 1977. Predation pressure and community structure of an intertidal soft bottom fauna. In: Keegan, B. F.; P. O. Céidigh \& P. J. S. Boaden, eds Biology of benthic organisms. New York, Pergamon Press, p. 513-519

SANTOS, S. L. \& SIMON, J. L. 1974. Distribution and abundance of the polychaetous annelids in a south Florida estuary. Bull. mar. Sci., 24:669-689.

SCHNEIDER, F.I. \& MANN, K. H. 1991. Species specific relationships of invertebrates to vegetation in a seagrass bed. I. Correlational studies. J. expl mar. Biol. Ecol., 145:101-117.

SHERIDAN, P. F. \& LIVINGSTON, R. J. 1983. Abundance and seasonality of infauna and epifauna inhabiting a Halodule wrightii meadow in Apalachicola Bay, Florida. Estuaries, 6:407-419. 
STONER, A. W. 1980. The role of seagrass biomass in the organization of benthic macrofaunal assemblages. Bull. mar. Sci., 30:537-551.

1983. Distributional ecology of amphipods and tanaidaceans associated with three sea grass species. J. Crust. Biol, 3:505-518.

SUGUIO, K. 1973. Introduçāo à sedimentologia. São Paulo, Edgard Blucher/EDUSP, 312 p.

SUMMERSON, H. C. \& PETERSON, C. H. 1984. Role of predation in organizing benthic communities of a temperate-zone seagrass bed. Mar. Ecol. Prog. Ser., 15:63-77.

TAKEDA, A. M. 1988. Estrutura de associações macrobênticas da Spartina alterniflora Loiseleur, 1807 no complexo estuarino-lagunar de Cananéia. Tese de doutorado. Universidade de São Paulo, Instituto Oceanografico, $70 \mathrm{p}$.

TEIXEIRA, C. 1979. Produção primária e algumas consideraçōes ecológicas da regiảo de Ubatuba (Lat. $23^{\circ} 30^{\prime} \mathrm{S}$-Long. $45^{\circ} 06^{\prime} \mathrm{W}$ ), Brasil. Bolm Inst. oceanogr., S Paulo, 28:23-28.

THISTLE, D. 1981. Natural physical disturbances and communities of marine soft bottoms. Mar. Ecol. Prog. Ser., 6:223-228.

VIRNSTEIN, R. H. 1987. Seagrass-associated invertebrate communities of the southeastern U.S.A.: a review. Florida mar. Res. Publs, 42:87-116.

; MIKKELSEN, P. S.; CAIRNS, $\mathrm{K}$. D. \& CAPONE, M. A. 1983. Seagrass beds versus sand bottoms: the trophic importance of their associated benthic invertebrates. Florida Sci., 46:363-381.
WELLS, F. E.; ROSE, R. A. \& LANG, S. 1985. An analysis of benthic marine invertebrate communities in subtidal seagrass and sand habitats in Shark Bay, Western Australia. Rec. West. Aust. Mus., 12:47-56.

WILLIAMS, A. B. 1984. Shrimps, lobsters, and crabs of the Atlantic coast of the eastern United States, Maine to Florida. Smithsonian Institute, 550 p.

WILSON, F.S. 1990 . Temporal and spatial patterns of settlement: a field study of molluscs in Bogue Sound, North Carolina. J. expl mar. Biol. Ecol., 139:201-220.

YOUNG, D. K. \& YOUNG, M. W. 1978. Regulation of species densities of seagrass-associated macrobenthos: evidence from field experiments in the Indian River estuary, Florida. J. Mar. Res., 36:569-593.

1982. Macrobenthic invertebrates in bare sand and seagrass (Thalassia testudinum) at Carrie Bow Cay, Belize. Smithsonian Contrib. mar. Sci., 12:115-126.

ZAR, J. H. 1984. Biostatistical analysis. 2nd ed. Englewood Cliffs, N. J. Prentice-Hall, 718 p.

(Manuscrito recebido 22 janeiro 1993; revisto 11 novembro 1994; aceito 22 dezembro 1994) 